\title{
Analysis of wntless (WLS) expression in gastric, ovarian, and breast cancers reveals a strong association with HER2 overexpression
}

Jonathan Stewart ${ }^{1,2}$, Jacqueline James ${ }^{1,2}$, Glenn W McCluggage ${ }^{1,2}$, Stephen McQuaid ${ }^{1,2}$, Kenneth Arthur ${ }^{1}$, David Boyle ${ }^{1,2}$, Paul Mullan ${ }^{3}$, Darragh McArt ${ }^{2}$, Benedict Yan $^{4}$, Gareth Irwin $^{3}$, D Paul Harkin ${ }^{3}$, Lei Zhengdeng ${ }^{5}$, Chee-Wee Ong ${ }^{1}$, Jia Yu ${ }^{5}$, David M Virshup ${ }^{5}$ and Manuel Salto-Tellez ${ }^{1,2,4}$

${ }^{1}$ Northern Ireland Molecular Pathology Laboratory, Centre for Cancer Research and Cell Biology, Queen's University, Belfast, Northern Ireland, UK; ${ }^{2}$ Tissue Pathology, Belfast Health and Social Care Trust, Belfast, Northern Ireland, UK; ${ }^{3}$ Breast \& Ovarian Cancer Programmes, Centre for Cancer Research and Cell Biology, Queens University Belfast, Belfast, Northern Ireland, UK; ${ }^{4}$ Cancer Science Institute of Singapore, National University Health System \& Yong Loo Lin School of Medicine, NUS, Singapore, Singapore and ${ }^{5}$ Cancer and Stem Cell Biology Program, Duke-National University of Singapore (NUS) Graduate Medical School,

Singapore, Singapore

The oncogenic role of WNT is well characterized. Wntless (WLS) (also known as GPR177, or Evi), a key modulator of WNT protein secretion, was recently found to be highly overexpressed in malignant astrocytomas. We hypothesized that this molecule may be aberrantly expressed in other cancers known to possess aberrant WNT signaling such as ovarian, gastric, and breast cancers. Immunohistochemical analysis using a TMA platform revealed WLS overexpression in a subset of ovarian, gastric, and breast tumors; this overexpression was associated with poorer clinical outcomes in gastric cancer $(P=0.025)$. In addition, a strong correlation was observed between WLS expression and human epidermal growth factor receptor 2 (HER2) overexpression. Indeed, $100 \%$ of HER2-positive intestinal gastric carcinomas, $100 \%$ of HER2-positive serous ovarian carcinomas, and $64 \%$ of HER2-positive breast carcinomas coexpressed WLS protein. Although HER2 protein expression or gene amplification is an established predictive biomarker for trastuzumab response in breast and gastric cancers, a significant proportion of HER2-positive tumors display resistance to trastuzumab, which may be in part explainable by a possible mechanistic link between WLS and HER2.

Modern Pathology (2015) 28, 428-436; doi:10.1038/modpathol.2014.114; published online 26 September 2014

The molecular mechanisms controlling the maturation and secretion of WNT proteins in the cells responsible for their production are attracting considerable attention. Wntless (WLS) (also known as GPR177 and Evi) was recently discovered as a key modulator of WNT protein secretion. ${ }^{1-3}$ This multipass transmembrane protein localizes to compartments of the secretory pathway including the Golgi apparatus, endosomes, and plasma membrane. WLS acts as a WNT cargo receptor, shuttling palmitoylated Wnts from the endoplasmic reticulum to the plasma membrane, and it is required for exocytosis of WNT

Correspondence: Professor M Salto-Tellez, MD (LMS), FRCPath, FRCPI, Centre for Cancer Research and Cell Biology, Queen's University Belfast, 97 Lisburn Road, Belfast BT9 7BL, Northern Ireland, UK.

E-mail: m.salto-tellez@qub.ac.uk

Received 5 May 2014; revised 20 July 2014; accepted 20 July 2014; published online 26 September 2014 proteins from the WNT-producing cells. ${ }^{4}$ In contrast to most other components of the WNT signaling pathway, only a single $W L S$ gene exists in vertebrate genomes. WLS appears to be required for the secretion of all WNT proteins in both the canonical and noncanonical WNT pathways. ${ }^{5}$

Augustin et $a l^{6}$ recently demonstrated that $W L S$ is highly overexpressed in malignant astrocytomas, indicating that the aberrant release of canonical and noncanonical WNT is a potential driver of glioma tumorigenesis required for proliferation, survival, and migration of glioma cells. Their work raises the question of whether $W L S$ has a similar role in other cancers known to have aberrant activation of WNT signaling, including ovarian, gastric, and breast cancers. $^{7-15}$

The aim of this study was to evaluate WLS protein expression, as well as the clinicopathological significance of this expression, in these three cancer types. 


\section{Materials and methods}

\section{Materials}

Primary tumors were studied from 198 patients with epithelial ovarian adenocarcinoma, representing all the main cancer subtypes, as well as full sections of paired primary tumors and omental metastases from 6 patients with serous ovarian tumors. All tumors were obtained from the Northern Ireland Biobank, under ethical approval (NIB12-0031). Tumors from 300 patients with breast cancer were also studied under appropriate ethical consent (NIB11-0017). Finally, 142 gastric tumors and 78 matched normal controls were analyzed, according to protocols described earlier ${ }^{16}$ and under ethical approval (National University of Singapore Institutional Review Board; NUS-IRB 06-063). These cases were selected randomly from retrospective collections in the pathology departments of the contributing institutions, reviewed by subspecialty pathologists in ovarian (GWMcC), breast (DB), and gastric (MS-T) cancer. All the immunohistochemical markers reported in this study were analyzed in a TMA framework, in the manner described below.

The available clinical and pathological data for the ovarian cancer study cohort included gender, age, tumor size, tumor stage, histological grade, survival, progression-free survival, and the chemotherapeutic regime and response. Scoring information for the same tumors was available for the baseline routine diagnostic immunohistochemical markers CA125, CK7, WT1, HMGA2, HNF1, PAX8, CK20, and CA19.9, chosen as they are relevant in routine histopathology reporting of ovarian cancer.

The available clinical and pathological data for the gastric cancer study cohort included age at surgery/treatment, tumor location, adenocarcinoma subtype, TNM stage, and tubular differentiation. Immunohistochemical scoring information for the same tumors was available for proteins HER2, SHH, PCNA, Ki67, CA IX, $\beta$-catenin, CD133, CD44, Mu, lgr5, EGFR, and p27Kip1, in the context of previous work in this tissue microarray. ${ }^{17}$

The available clinical and pathological data for the breast cancer study cohort included age at diagnosis, gender, TNM stage, histological subtype, lymph node involvement, and lymphovascular invasion. Scoring information for the same tumors was available for the routine diagnostic immunohistochemical markers estrogen receptor (ER), progesterone receptor (PR), and human epidermal growth factor receptor 2 (HER2) as part of an ongoing study.

\section{Methods}

Tissue microarray construction. Three sets of ovarian cancer tissue microarrays containing three cores from 198 primary ovarian cancer cases, three sets of breast tissue microarrays containing three cores from 300 breast cancer cases, and one set of gastric tissue mircroarrays containing 142 gastric cancer cases were constructed as detailed in previous studies. ${ }^{18,19}$ A 1.0-mm-diameter needle was used to punch a donor core from morphologically representative areas of a donor tissue block. The core was subsequently inserted into a recipient paraffin block using a MTA-1 Manual Tissue Arrayer (Beecher Instruments, WI). Three cores were taken from the center of the tumor tissue. A single core was taken from histologically normal ovary. Consecutive tissue microarray sections of $3.5-\mathrm{mm}$ thickness were cut and placed on slides for immunohistochemical analysis.

Immunohistochemistry. The primary antibody was

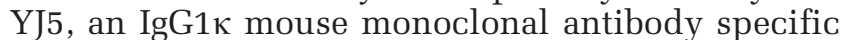
for human WLS/GPR177 (Coombs et $a l^{4}$ and available from Millipore, cat. \# MABS87). Human breast cancer cell line MDA-231 with knockdown of WLS expression and HeLa cell lines with constitutive WLS expression were fixed in formalin and embedded in paraffin (mimicking the laboratory conditions of our clinical samples), and used for immunohistochemistry optimization. Numerous antigen dilutions, antigen retrieval methods, and antigen exposure times were tested until the adequate expression patterns were detected in the cell line controls, namely no expression in the knockdown cells and predominant cytoplasmic expression in the HeLa cells (Figure 1). Negative

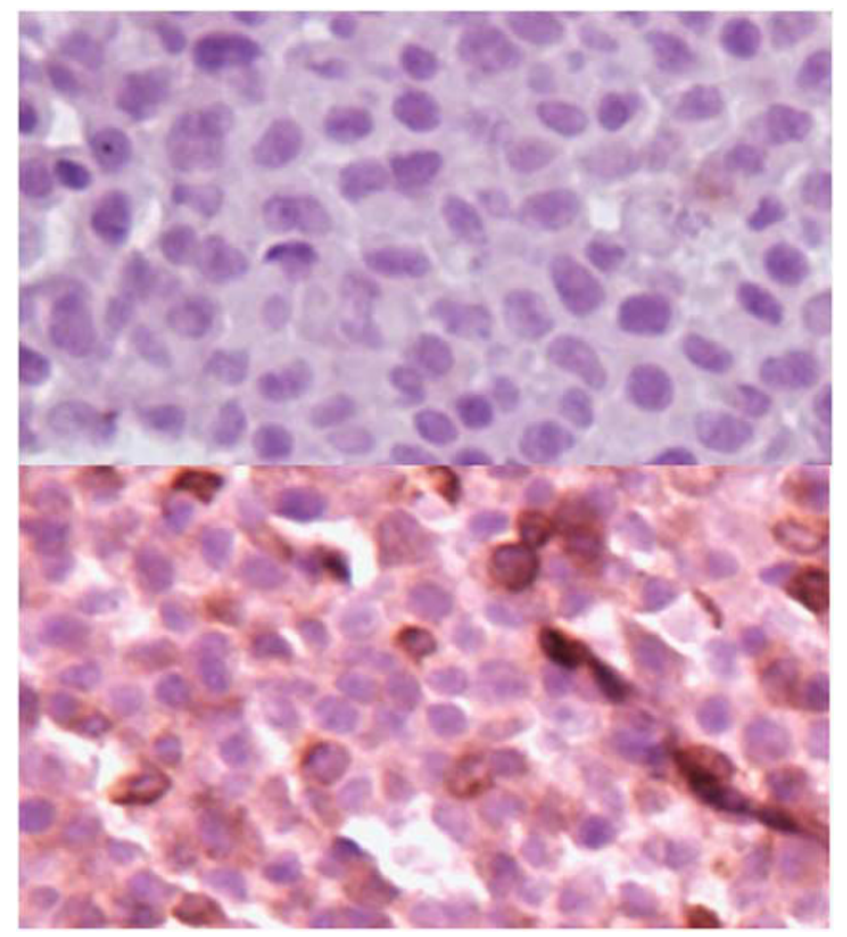

Figure 1 WLS expression in MDA-231 with WLS knockdown (above) and HeLa cells with constitutively expressed WLS (below), magnification $\times 63$. 
controls consisted of the omission of primary antibody without any other changes to subsequent procedures. Once optimized, the antibody was tested in numerous normal tissues. It was observed that there was consistent cytoplasmic expression in the myoepithelial constitutive layer of normal breast duct epithelium, which from that point onward represented our positive control for further experiments. The optimized method was as follows. Antigen retrieval was performed in a microwave for 20 mins in citrate buffer. Endogenous peroxidase activity was blocked using a solution of $10 \%$ hydrogen peroxide (dissolved in 1\% alcohol). Sections were washed and incubated with WLS primary antibody at a dilution of 1:100 (dissolved in TBS) for $24 \mathrm{~h}$ at $4{ }^{\circ} \mathrm{C}$. Immunohistochemical staining was performed using the peroxidase EnVision kit (DakoCytomation, Copenhagen, Denmark) according to the manufacturer's instructions. Sections were counterstained with Mayers Haematoxylin (Leica CV5030 Autostainer).

For HER2 immunohistochemical analysis, the ORACLE HER2 Bond IHC system (CB11) mouse monoclonal antibody (Bond-maX, Menarini, Florence, Italy) was also used. HER2 immunohistochemical staining was performed using the automated Leica Microsystems Bondmax (Leica, Wetzlar, Germany) according to the manufacturer's instructions. ${ }^{20}$ Sections were then lightly counterstained with hematoxylin.

Tissue microarray scoring. Immunohistochemical staining was scored either microscopically or using the tissue microarray digital pathology toolbox (PathXL). The intensity of tumor cell staining was scored semiquantitatively on a 4-point scale $(0=$ unstained at high power, $1=$ visible at high power but not at low power, $2=$ visible at low power, and $3=$ strongly visible at low power). The proportion of tumor cells with positive staining was scored semiquantitatively on a 5 -point scale $(1=0-20 \%$, $2=21-40 \%, 3=41-60 \%, 4=61-80 \%$, and $5=81-$ $100 \%)$. The score for each core is calculated by multiplying the intensity by proportion. Owing to the natural dropout of samples in tissue microarray studies, the number of optimal ovarian cancer cores was reduced from a maximum possible 594 to 526; gastric cancer cores were reduced from 142 to 100; and breast cancer cores were reduced from 900 to 563 .

Statistical analysis and clinicopathological correlation. Correlation analysis performed for the triplicate sets of all tissue microarrays indicated consistency of expression across the sets, proving the suitability of tissue microarrays for the analysis proposed. WLS expression was compared between tumor subgroups using Mann-Whitney $U$-tests (as used previously by Cavicchioli et $a l^{21}$ ) and chisquared analysis. The association between WLS scoring and clinicopathological parameters was analyzed using chi-squared analysis, and the correlation between WLS scoring and scoring for baseline markers was analyzed using Spearman's rank correlation. Kaplan-Meier survival analysis was used to compare survival in patients with WLSpositive versus WLS-negative tumors. Statistical significance was calculated by two-tailed statistical analysis. In the figures and tables, a single asterisk marks a $P$-value $<0.05$, two asterisks represent $P$-values $<0.01$, and three asterisks represent $P$-values $<0.001$.

\section{Results}

\section{WLS Protein Expression in Cancer}

Ovary. Normal ovarian surface epithelium showed no WLS expression (Figure 2a). WLS expression varied significantly between ovarian cancer pathological subtypes $\left({ }^{* * *} P<0.001\right)$. In all, 83\% (19/23) of endometrioid carcinomas, $70 \%(95 / 136)$ of serous carcinomas, $69 \%$ (9/13) of mucinous carcinomas, and $48 \%(14 / 29)$ of clear cell carcinomas showed WLS expression. All four ovarian tumor subtypes had significantly higher WLS expression than normal ovarian surface layer: serous ${ }^{* * *} P<0.001$, clear cell ${ }^{* *} P=0.004$, endometrioid ${ }^{* * *} P<0.001$, and mucinous ${ }^{* *} P=0.002$. When WLS expression was compared between subtypes, it was found to be significantly higher in endometrioid compared with serous $\left({ }^{*} P=0.039\right)$ and clear cell $\left.{ }^{* *} P=0.010\right)$ carcinomas, and higher, although not significantly, than mucinous carcinoma (possibly because of the small sample size of mucinous carcinomas). Although serous ovarian cancer was initially analyzed as a single disease entity, recent molecular studies suggest that high-grade serous ovarian cancer should be treated as a separate disease from low-grade and borderline serous ovarian cancer. ${ }^{22}$ Indeed, WLS expression varied significantly between serous ovarian cancer grades $\left({ }^{* * *} P<0.001\right)$. WLS was found to be expressed in $77 \%(89 / 116)$ of high-grade serous tumors compared with $25 \%(5 / 20)$ of borderline and low-grade serous tumors. Highgrade serous carcinomas expressed significantly higher levels of WLS than both low-grade serous $\left({ }^{* *} P=0.004\right)$ and borderline serous $\left({ }^{* *} P=0.002\right)$ tumors. Metastatic cases showed significantly higher expression than both borderline $\left({ }^{* *} P=0.002\right)$ and low-grade carcinomas $\left({ }^{* *} P=0.003\right)$, and higher, although not significantly, than high-grade serous tumors $(P=0.064)$. Increasing WLS expression with histological grade is shown in Figure 3.

Gastric. Figures 2e-h show representative images of WLS expression in the two main gastric cancer subtypes (intestinal and diffuse), intestinal metaplasia, and normal epithelium. WLS expression varied significantly between gastric cancer subtypes $\left({ }^{* * *} P=<0.001\right)$. WLS expression was found in $20 \%$ 


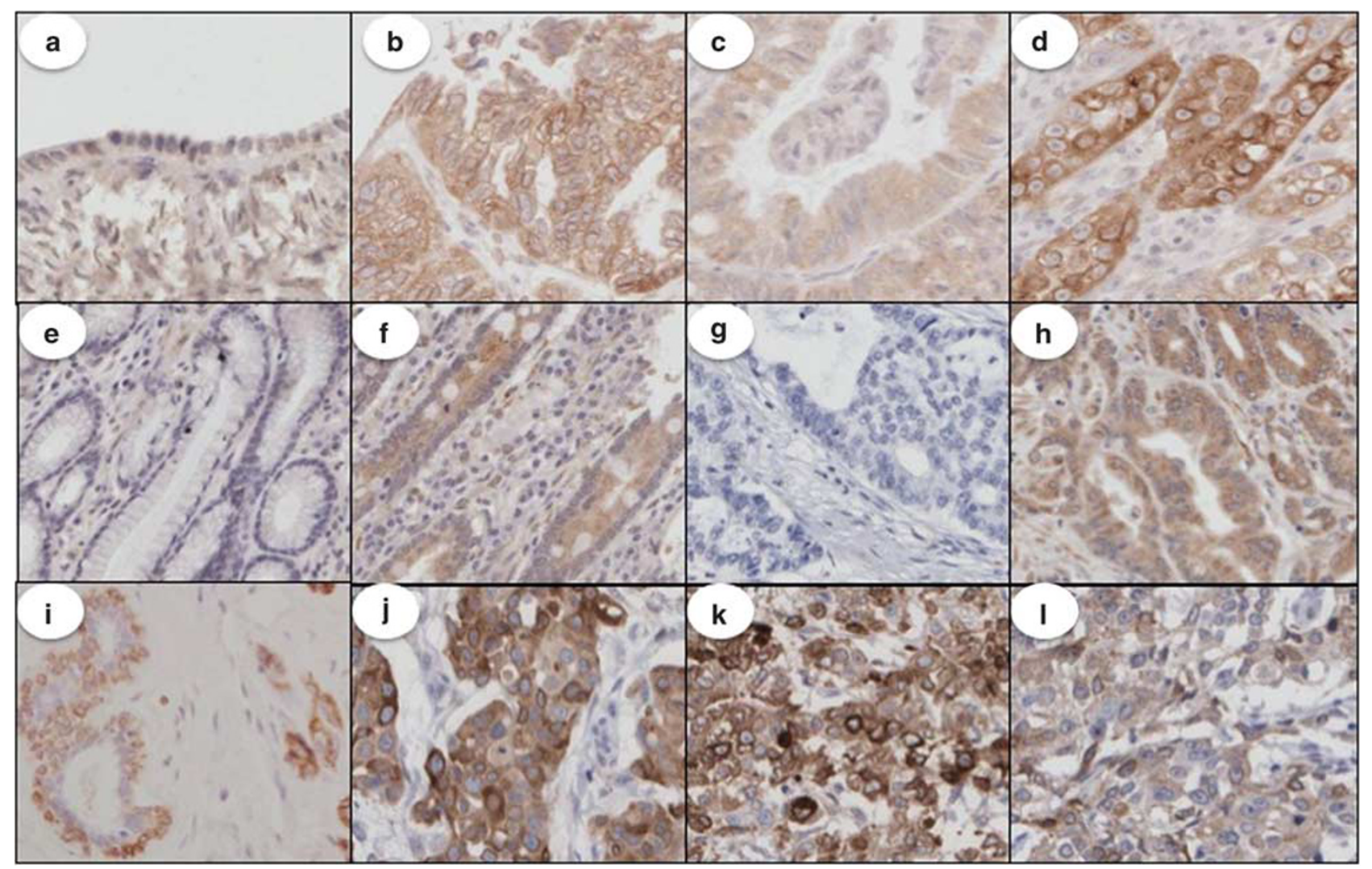

Figure 2 Representative images of WLS expression in ovarian, gastric, and breast carcinomas (all at magnification $\times 40$ ). Demonstration of lack of WLS protein expression by immunohistochemistry in normal ovarian surface lining (a), and variable expression in endometrial (b), mucinous (c), and clear cell (d) ovarian cancers $(P<0.001)$. Demonstration of lack of expression in normal gastric epithelium and in diffuse-type gastric cancer (e and $\mathbf{g}$ ), whereas intestinal metaplasia and intestinal-type gastric cancer show enhanced expression (f and $\mathbf{h}$ ) $(P<0.001)$. Demonstration of the myoepithelial component of normal breast duct expressing WLS (i). We found no significant difference in WLS expression between breast cancer subtypes (see $\mathbf{j}-\mathbf{l}$, representing ductal, lobular and mixed ductal-lobular breast cancer).

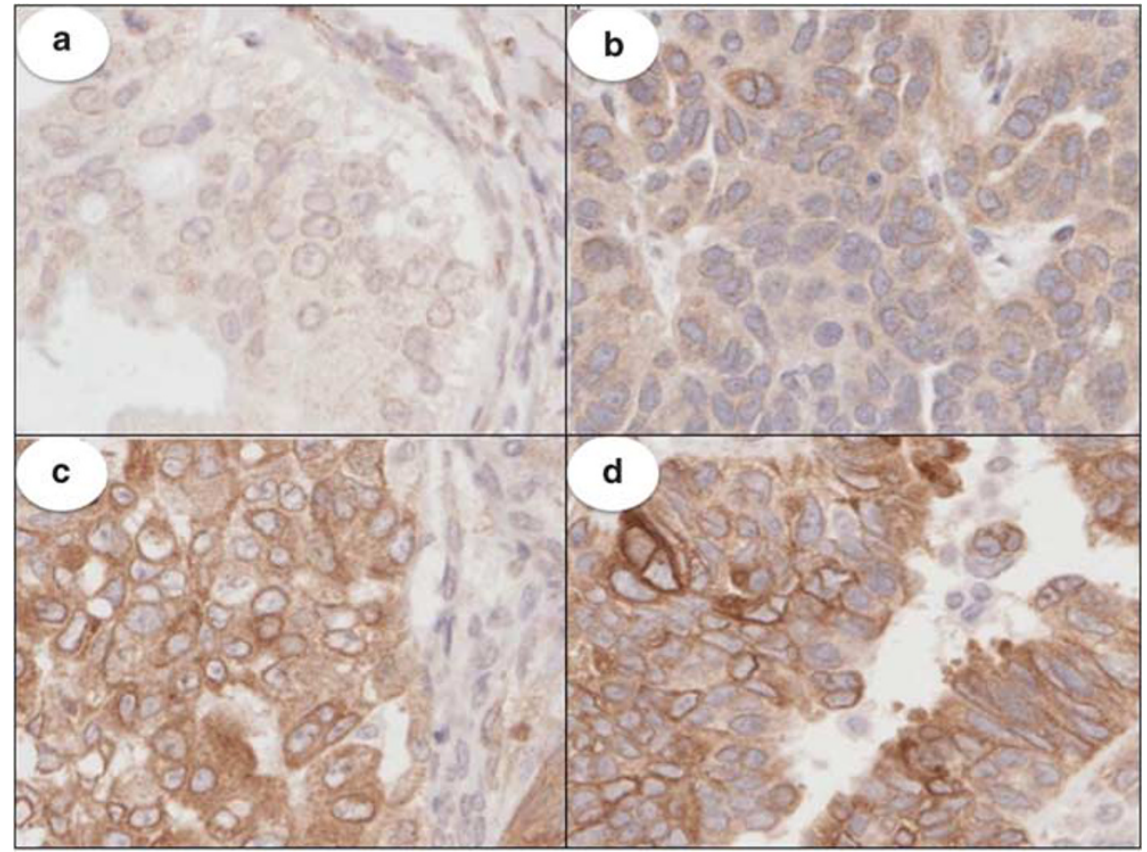

Figure 3 Morphomolecular progression of WLS expression in serous ovarian cancer, from borderline (a), low-grade (b), high-grade (c), and metastatic $(\mathbf{d})$ serous carcinomas (all at magnification $\times 40$ ). 
$(1 / 5)$ of mixed gastric tumors, $45 \%(9 / 20)$ of diffuse gastric tumors, and $61 \%(42 / 69)$ of intestinal gastric tumors. Diffuse $\left({ }^{*} P=0.012\right)$ and intestinal $\left.{ }^{* * *} P=<0.001\right)$ gastric tumors expressed significantly higher levels of WLS than normal gastric epithelium. Mixed gastric tumors showed higher WLS expression compared with matched normal samples, although not significantly. Intestinal-type gastric tumors showed higher WLS expression than both diffuse and mixed gastric tumors.

Breast. In this study, 48\% (109/226) of breast cancer cases exhibited WLS protein overexpression. Unlike in ovarian and gastric carcinomas, no significant difference in WLS expression was found between the different histological subtypes of breast cancer. A similar proportion of each of the three main histological subgroups (invasive ductal carcinoma, invasive lobular carcinoma, and mixed tumors) expressed WLS. See Figures 2i-l.

\section{Correlation of WLS Expression with Clinicopathological Parameters and Other Cancer Markers}

Ovary. Clinicopathological data were only available for serous carcinomas. Chi-squared analysis revealed that only the degree of differentiation was significantly associated with WLS expression $\left.{ }^{* * *} P=<0.001\right)$, as previously mentioned. KaplanMeier survival analysis comparing WLS expression with overall survival and progression-free survival showed no significant differences between WLSpositive and WLS-negative tumors. When WLS expression was compared with the expression of other cancer markers, a strong negative correlation with CA125 expression $\left({ }^{*} P=0.002\right)$ and a strong positive correlation with HMGA2 expression was found $\left({ }^{* * *} P<0.001\right)$. In all, $4 \%(7 / 201)$ of epithelial ovarian carcinoma tumor samples were HER2 positive. A significant positive correlation was found between WLS scoring and HER2 expression $\left({ }^{* *} P=0.001\right)$. All the HER2-positive ovarian carcinomas $(7 / 7,100 \%)$ expressed WLS, as shown in Figures $5 \mathrm{a}$ and $\mathrm{d}$. Five serous ovarian carcinomas were HER2 positive. When the correlation between HER2 scoring and WLS scoring was analyzed for serous ovarian carcinomas alone, this significant correlation remained $\left({ }^{* * *} P<0.001\right)$. All the HER2positive serous ovarian carcinomas $(5 / 5,100 \%)$ expressed WLS.

Gastric. $\chi^{2}$ analysis revealed no significant relationship between WLS expression and any clinicopathological parameter. Kaplan-Meier survival analysis showed a nonsignificant trend toward poorer overall survival and progression-free survival in WLS-positive gastric cancer patients. As intestinal gastric carcinomas showed the highest WLS expression, survival was also analyzed for intestinal tumors alone. Progression-free survival was significantly shorter in WLS-positive intestinal gastric tumors $\left(P=0.025^{*}\right)$ Figure $4 \mathrm{~b}$; overall survival was also shorter, albeit not significantly (Figure 4a).

WLS expression showed a significant correlation with other cancer markers, including HER2 genomic copy number status (HER2 FISH and CISH are 100\% concordant in our earlier gastric cancer study) and Ki67 ( $\left.{ }^{*} P=0.031\right)$ expression. When limited to only intestinal gastric carcinomas, WLS expression correlated significantly with HER2 genomic copy number status ( $\left.{ }^{*} P=0.010\right)$ and immunohistochemical expression $\left({ }^{*} P=0.041\right)$. When the relationship between WLS expression and HER2 expression was analyzed further using chi-squared analysis, 100\% (8/8) of HER2-positive intestinal gastric carcinomas expressed WLS $\left({ }^{*} P=0.020\right)$. This is illustrated in Figures $5 \mathrm{~b}$ and e.

Breast. $\quad \chi^{2}$ analysis revealed no statistically significant association between WLS expression and any clinicopathological parameter. The association of WLS expression and histological grade approached statistical significance $(P=0.068)$. The proportion of breast tumors expressing WLS increased with increasing grade. There was no survival difference between WLS-positive and WLS-negative breast carcinoma patients. WLS expression showed a significant positive correlation with HER2 expression $\left({ }^{* *} P=0.001\right)$ Figures $5 \mathrm{c}$ and $\mathrm{f}$, and a significant negative correlation with ER $\left({ }^{*} P=0.015\right)$ and $P R$ $\left.{ }^{*} P=0.034\right)$ expression.

\section{Discussion}

\section{WLS Expression in Ovarian, Gastric, and Breast Cancers}

The involvement of WNT signaling in ovarian, gastric, and breast cancers is well characterized. ${ }^{23,24}$

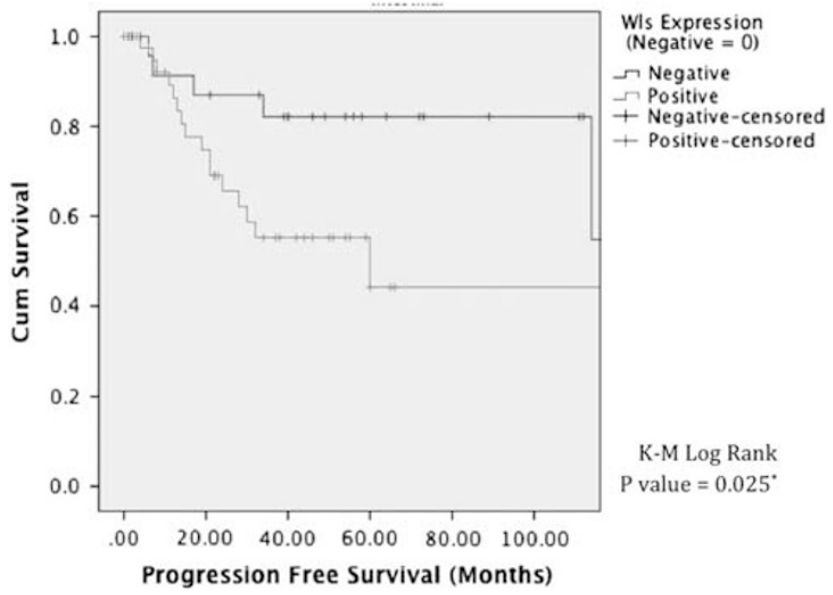

Figure 4 Relationship of WLS expression with progression-free survival (a) and overall survival (b) in intestinal-type gastric cancer. 


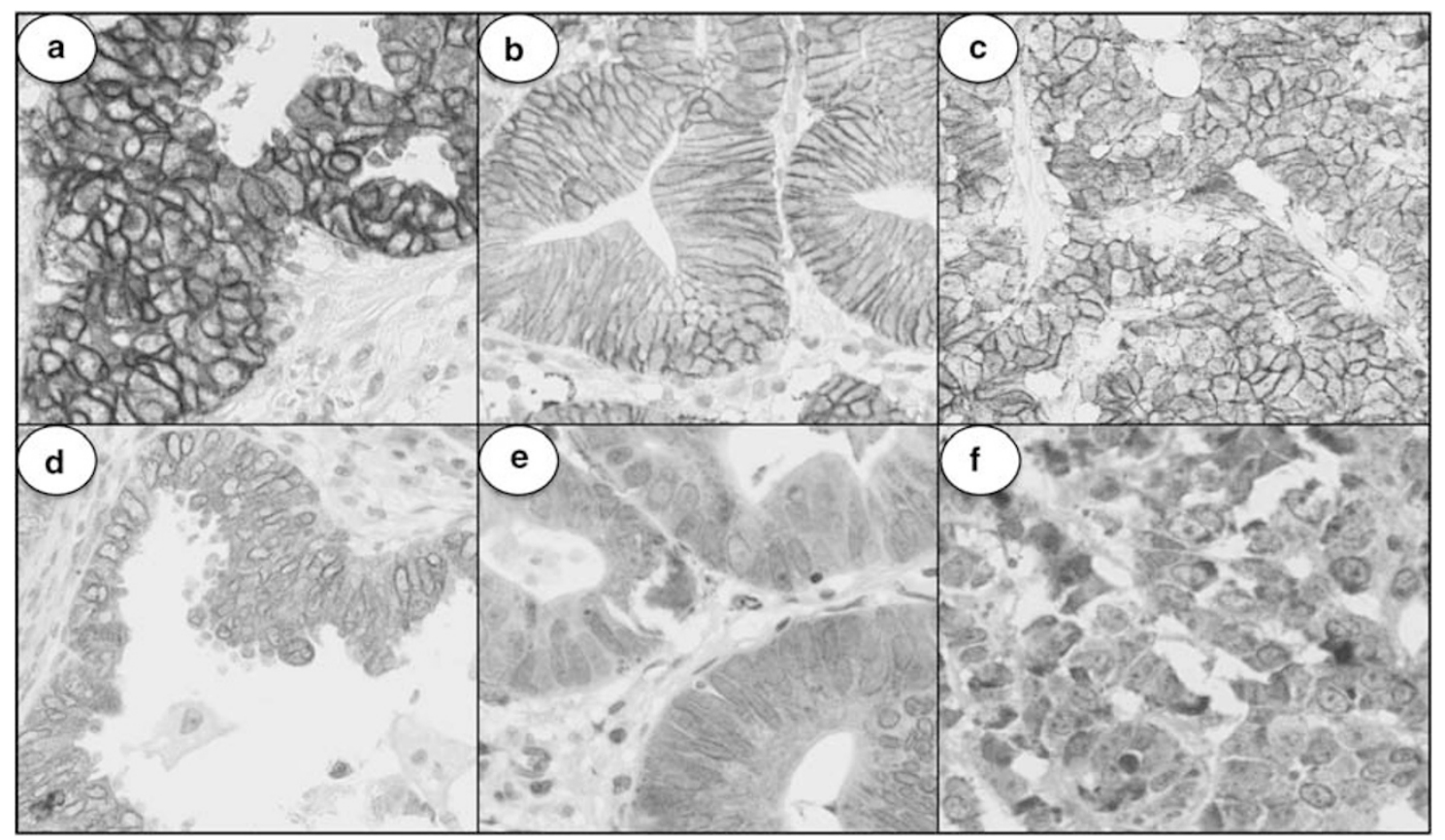

Figure 5 Relationship between WLS and HER for ovarian, gastric, and breast carcinomas. HER2 protein expression in high-grade ovarian serous, intestinal gastric, and breast cancers (a-c, respectively), and WLS expression for the same cancer types (d-f, respectively).

The finding that WLS is overexpressed in a subset of ovarian, gastric, and breast cancers provides further evidence for the role of WNT signaling in these malignancies. Our finding that significantly higher numbers of endometrioid carcinomas strongly express WLS compared with the other subtypes is consistent with previous findings that activation of WNT signaling is more common in this subtype. ${ }^{25}$ The finding that significantly higher numbers of high-grade serous tumors strongly express WLS compared with low-grade and borderline serous tumors highlights the possible oncogenic role of WNT in these cancers. In addition, WLS expression was much more common in intestinal gastric tumors as compared with diffuse and mixed gastric tumors, consistent with the known activation of WNT signaling, as seen by nuclear expression of $\beta$-catenin, in this cancer subtype. ${ }^{11,26,27}$ Furthermore, the percentage of breast cancers with WLS overexpression reported in this study, $48 \%$, is consistent with the overexpression of components of the WNT signaling pathway reported earlier, and reviewed by Howe and Brown. ${ }^{24}$

\section{HER2 Expression in Gastric, Ovarian, and Breast Cancers}

Since the discovery of HER2 amplification in human breast cancer 25 years ago, ${ }^{28}$ HER2 overexpression and gene amplification have been identified in other cancer types including ovarian and gastric cancer. ${ }^{29}$ The association of HER2 expression with poor prognosis in breast, ${ }^{30}$ gastric, ${ }^{31-34}$ and ovarian cancers $^{35}$ has been reported, although, to the best of our knowledge, the only 'well-defined' association with prognosis is with breast cancer. HER2 is also an important predictive biomarker for trastuzumab response in breast ${ }^{36,37}$ and gastric cancers. ${ }^{38}$ The value of HER2 as a predictive biomarker in ovarian cancer is less established. HER2 has been targeted in ovarian cancer in the clinical trial setting, and preclinical data indicate that HER2 inhibitors including trastuzumab, ${ }^{39}$ lapatinib, ${ }^{40-42}$ and pertuzumab $^{43,44}$ are effective in a subset of HER2positive ovarian tumors, although this is still not the standard of care for this malignancy.

\section{WLS and HER2 in Gastric, Ovarian, and Breast Cancers}

Interestingly, when we compared WLS expression with the expression of a panel of cancer markers, a strong correlation was observed between WLS expression and HER2 expression across cancer subtypes (Figure 5) (Table 1). In all, 100\% (8/8) of HER2-positive intestinal gastric carcinomas, $100 \%$ (5/5) of HER2-positive serous ovarian carcinomas, and $64 \%$ (41/64) of HER2-positive breast carcinomas expressed WLS. This finding has clear biological and clinical implications.

Biologically, the increased or nuclear expression of $\beta$-catenin has been used in the literature as a marker for WNT signaling activation in general, as well as a means to establish a possible relation 
Table 1 Correlation of WLS scoring and HER2 scoring for serous ovarian, intestinal gastric, and breast carcinomas

\begin{tabular}{|c|c|c|c|c|c|}
\hline & $\mathrm{N}$ & Pearson correlation & $\mathrm{P}$-value & Spearman's rank correlation & P-value \\
\hline Serous ovarian cancer & 131 & 0.328 & $0.000^{* * *}$ & 0.148 & 0.091 \\
\hline Intestinal gastric cancer & 57 & 0.27 * & 0.041 * & 0.276 & $0.038^{*}$ \\
\hline breast cancer & 217 & 0.200 & $0.003^{* *}$ & 0.218 & $0.001^{* *}$ \\
\hline
\end{tabular}

between WNT signaling and HER2 expression. ${ }^{45-48}$ However, $\beta$-catenin protein expression in cancer is controversial, as the presence and location of expression is often inconsistent between studies. As only one WLS gene exists in the human genome that is responsible for the secretion of all WNT proteins, WLS may represent a plausible alternative to $\beta$-catenin as a measure of WNT signaling activation. Clinically, the observation reported here may be of particular relevance. HER2 is an established poor prognostic marker in breast ${ }^{30}$ cancer, and several studies also report it as a poor prognostic marker in gastric ${ }^{31-33}$ cancer. In this study, WLS expression was also associated with significantly shorter progression-free survival in gastric cancer cases $\left({ }^{*} P=0.025\right)$. A mechanistic link between HER2 and WLS may help explain the aggressive and chemoresistant nature of HER2positive breast, ovarian, and gastric cancer tumors, and why patient with these tumors have lower than expected response rate to agents that target HER2. Recent literature suggests a possible pathway in which WLS and HER2 may be involved via the transcription factor signal transducer and activator of transcription 3 (STAT3) and the known mediator of trastuzumab and chemotherapy resistance nuclear factor kappa-light-chain-enhancer of acti-

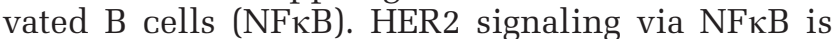
known to contribute to progression and chemoresistance in ovarian, ${ }^{49-51}$ gastric, ${ }^{52}$ and breast cancers. ${ }^{53-56}$ In primary breast tumors, HER2 not only stimulates $\mathrm{NF} \kappa \mathrm{B}$ expression but is also itself unregulated by $\mathrm{NF} \kappa \mathrm{B}$, stimulating a closed loop responsible for chemoresistance, which includes STAT3 ${ }^{54-56}$ WLS may be another key component of this signaling pathway. In this way, WLS may act as a biomarker to identify patients who are unlikely to respond to chemotherapy and/or trastuzimab alone, and join HER2 analysis in the armamentarium of personalized medicine-related biomarkers.

WLS is also a potential therapeutic target. ${ }^{57}$ Only $20-30 \%$ of HER2-positive breast cancer patients respond to trastuzumab monotherapy. ${ }^{58,59}$ If WLS is a key component of a pathway responsible for trastuzumab resistance that is expressed across cancer subtypes, then WLS could be targeted therapeutically alone, or in conjunction with trastuzimab, to help overcome resistance.

In conclusion, this study is the first characterization of the protein expression of the WNT secretion protein WLS in ovarian, gastric, and breast cancers.
This study has revealed a strong association between the expression of WLS and HER2 that has important biological and clinical implications. HER2 is an important predictive biomarker to identify patients who likely to respond to trastuzumab. However, the response rate of HER2-positive patients to this therapy remains relatively low. Recent literature in combination with the findings of this study suggests that WLS may be a key component of a pathway responsible for trastuzumab resistance. WLS would represent a very attractive therapeutic target, especially as it is expressed across cancer subtypes. Further investigation may provide concise mechanistic insights into the association between these important signaling molecules.

\section{Disclosure/conflict of interest}

The authors declare no conflict of interest.

\section{References}

1 Bänziger C, Soldini D, Schütt C, et al. Wntless, a conserved membrane protein dedicated to the secretion of Wnt proteins from signaling cells. Cell 2006;125:509-522.

2 Bartscherer K, Pelte N, Ingelfinger D, et al. Secretion of Wnt ligands requires Evi, a conserved transmembrane protein. Cell 2006;125:523-533.

3 Belenkaya TY, Wu Y, Tang X, et al. The retromer complex influences Wnt secretion by recycling Wntless from endosomes to the trans-golgi network. Dev Cell 2008;14:120-131.

4 Coombs GS, Yu J, Canning CA, et al. WLS-dependent secretion of WNT3A requires Ser209 acylation and vacuolar acidification. J Cell Sci 2010;123:3357-3367.

5 Najdi R, Proffitt K, Sprowl S, et al. A uniform human Wnt expression library reveals a shared secretory pathway and unique signaling activities. Differentiation 2012;84:203-213.

6 Augustin I, Goidts V, Bongers A, et al. The Wnt secretion protein Evi/Gpr177 promotes glioma tumorigenesis. EMBO Mol Med 2012;4:38-51.

7 Bafico A, Liu G, Goldin L, et al. An autocrine mechanism for constitutive Wnt pathway activation in human cancer cells. Cancer Cell 2004;6:497-506.

8 Rask K, Nilsson A, Brannstrom M, et al. Wnt-signalling pathway in ovarian epithelial tumors: increased expression of beta-catenin and GSK3beta. Br J Cancer 2000;89:1298-1304. 
9 Badiglian Filho L, Oshima C, De Oliveira-Lima F, et al. Canonical and non canonical Wnt pathway: a comparison among normal ovary, benign ovarian tumor and ovarian cancer. Oncol Rep 2009;21:313-320.

10 Nakatsuru S, Yanagisawa A, Ichii S, et al. Somatic mutation of the APC gene in gastric cancer: frequent mutations in very well differentiated adenocarcinoma and signet-ring cell carcinoma. Hum Mol Genet 1992;1:559-563.

11 Ebert MPA, Fei G, Kahmann S, et al. Increased $\beta$ catenin mRNA levels and mutational alterations of the APC and $\beta$-catenin gene are present in intestinal-type gastric cancer. Carcinogenesis 2002;23:87-91.

12 Pan K, Liu W, Zhang l, et al. Mutations in components of the Wnt signaling pathway in gastric cancer. World J Gastroentero 2008;14:1570-1574.

13 Lin S, Xia W, Wang JC, et al. $\beta$-Catenin, a novel prognostic marker for breast cancer: Its roles in cyclin D1 expression and cancer progression. Proc Natl Acad Sci USA 2000;97:4262-4266.

14 Schlosshauer PW, Brown SA, Eisinger K, et al. APC truncation and increased $\beta$-catenin levels in a human breast cancer cell line. Carcinogenesis 2000;21: 1453-1456.

15 Jönsson $\mathrm{M}$, Borg $\AA$, Nilbert $\mathrm{M}$, et al. Involvement of adenomatous polyposis coli (APC)/ $\beta$-catenin signalling in human breast cancer. Eur J Cancer 2000;36: 242-248.

16 Yan B, Yau EX, Bte Omar SS, et al. A study of HER2 gene amplification and protein expression in gastric cancer. J Clin Pathol 2010;63:839-842.

17 Wang T, Ong CW, Shi J, et al. Sequential expression of putative stem cell markers in gastric carcinogenesis. Br J Cancer 2011;105:658-665.

18 Das K, Mohd Omar M, Ong C, et al. TRARESA: a tissue microarray-based hospital system for biomarker validation and discovery. Pathology 2008;40:441-449.

19 Salto-Tellez M, Nga ME, Han HC, et al. Tissue microarrays characterise the clinical significance of a VEGF-A protein expression signature in gastrointestinal stromal tumors. Br J Cancer 2007;96:776-782.

20 Fassina A, Cappellesso R, Guzzardo V, et al. Epithelialmesenchymal transition in malignant mesothelioma. Mod Pathol 2012;25:86-99.

21 Cavicchioli Buim ME, Gurgel CAS, Gonçalves Ramos EA, et al. Activation of sonic hedgehog signaling in oral squamous cell carcinomas: a preliminary study. Hum Pathol 2011;42:1484-1490.

22 Prat J. Ovarian Carcinomas: Five distinct disease with different origins, genetic alterations and clinicopatholoigcal features. Virchows Arch 2012;460:237-249.

23 Gatcliffe T, Monk B, Planutis K, et al. Wnt signaling in ovarian tumorigenesis. Int J Gynecol Cancer 2008;18: 954-962.

24 Howe L, Brown A. Wnt signaling and Breast Cancer. Cancer Biol Ther 2004;3:36-41.

25 Wright $\mathrm{K}$, Wilson $\mathrm{P}$, Morland S, et al. Beta-Catenin mutation and expression analysis in ovarian cancer: Exon 3 mutations and nuclear translocation in 16\% of endometrioid tumors. Int J Cancer 1999;82:625-629.

26 Ebert MPA, Yu J, Hoffmann J, et al. Loss of BetaCatenin Expression in Metastatic Gastric Cancer. J Clin Oncol 2003;21:1708-1714.

27 Ogasawara N, Tsukamoto T, Mizoshita T, et al. Mutations and nuclear accumulation of Beta-catenin correlate with intestinal phenotypic expression in human gastric cancer. Histopathology 2006;49:612-621.
28 van de Vijver MJ, Peterse JL, Mooi WJ, et al. NeuProtein Overexpression in Breast Cancer. N Engl J Med 1988;319:1239-1245.

29 Gutierrez C, Schiff R. HER2: biology, detection and clinical implications. Arch Pathol Lab Med 2011;135: $55-62$.

30 Tandon AK, Clark GM, Chamness GC, et al. HER-2/neu oncogene protein and prognosis in breast cancer. J Clin Oncol 1989;7:1120-1128.

31 Uchino S, Tsuda H, Maruyama K, et al. Overexpression of c-erbB-2 protein in gastric cancer. Its correlation with long-term survival of patients. Cancer 1993;72: 3179-3184.

32 Mizutani T, Onda M, Tokunaga A, et al. Relationship of C-erbB-2 protein expression and gene amplification to invasion and metastasis in human gastric cancer. Cancer 1993;72:2083-2088.

33 Nakajima M, Sawada H, Yamada $\mathrm{Y}$, et al. The prognostic significance of amplification and overexpression of c-met and c-erb B-2 in human gastric carcinomas. Cancer 1999;85:1894-1902.

34 Jørgensen J, Hersom M. HER2 as a prognostic marker in gastric cancer - a systematic analysis of data from the literature. J Cancer 2012;3:137-144.

35 Berchuck A, Kamel A, Whitaker R, et al. Overexpression of HER-2/neu is associated with poor survival in advanced epithelial ovarian cancer. Cancer Res 1990;50:4087-4091.

36 Baselga J, Norton L, Albanell J, et al. Recombinant humanized anti-HER2 antibody (Herceptin ${ }^{\mathrm{TM}}$ ) enhances the antitumor activity of paclitaxel and doxorubicin against HER2/neu overexpressing human breast cancer xenografts. Cancer Res 1998;58:2825-2831.

$37 \mathrm{Yu} \mathrm{D}$, Hung M. Overexpression of ErbB2 in cancer and ErbB2-targetting strategies. Oncogene 2000;19: 6115-6121.

38 Bang Y, Van Cutsem E, Feyereislova A, et al. Trastuzumab in combination with chemotherapy versus chemotherapy alone for treatment of HER2-positive advanced gastric or gastro-oesophageal junction cancer (ToGA): a phase 3, open-label, randomised controlled trial. Lancet 2010;376:687-697.

39 Bookman MA, Darcy KM, Clarke-Pearson D, et al. Evaluation of monoclonal humanized anti-HER2 antibody, trastuzumab, in patients with recurrent or refractory ovarian or primary peritoneal carcinoma with overexpression of HER2: a phase II trial of the gynecologic oncology group. J Clin Oncol 2003;21:283-290.

40 Kimball KJ, Numnum TM, Kirby TO, et al. A phase I study of lapatinib in combination with carboplatin in women with platinum sensitive recurrent ovarian carcinoma. Gynecol Oncol 2008;111:95-101.

41 Rivkin SE, Muller C, Malmgren JA, et al. A phase I/II study of lapatinib plus carboplatin and paclitaxel in relapsed ovarian and breast cancer. Clinical Ovarian Cancer 2009;2:112-117.

42 Weroha SJ, Oberg AL, Ziegler KLA, et al. Phase II trial of lapatinib and topotecan (LapTop) in patients with platinum-refractory/resistant ovarian and primary peritoneal carcinoma. Gynecol Oncol 2011;122:116-120.

43 Gordon MS, Matei D, Aghajanian C, et al. Clinical activity of pertuzumab (rhuMAb 2C4), a HER dimerization inhibitor, in advanced ovarian cancer: potential predictive relationship with tumor HER2 activation status. J Clin Oncol 2006;24:4324-4332.

44 Makhija S, Amler LC, Glenn D, et al. Clinical activity of gemcitabine plus pertuzumab in platinum-resistant 
ovarian cancer, fallopian tube cancer, or primary peritoneal cancer. J Clin Oncol 2010;28:1215-1223.

45 López-Knowles E, Zardawi SJ, McNeil CM, et al. Cytoplasmic localization of $\beta$-catenin is a marker of poor outcome in breast cancer patients. Cancer Epidemiol Biomarkers Prev 2010;19:301-309.

46 Ni M, Chen Y, Lim E, et al. Targeting androgen receptor in estrogen receptor-negative breast cancer. Cancer Cell 2011;20:119-131.

47 Geyer FC, Lacroix-Triki M, Savage K, et al. BetaCatenin pathway activation in breast cancer is associated with triple-negative phenotype but not with CTNNB1 mutation. Mod Pathol 2011;24:209-231.

48 Khalil S, Tan GA, Giri DD, et al. Activation status of Wnt/ß-catenin signaling in normal and neoplastic breast tissues: relationship to HER2/neu expression in human and mouse. PLoS One 2012;7:33421.

49 White K, Rider D, Kalli KR, et al. Genomics of the NF- $\mathrm{B}$ signaling pathway: hypothesized role inovarian cancer. Cancer Causes Control 2011;22:785-801.

50 Yang P, Lorenowicz MJ, Silhankova M, et al. Wnt Signaling Requires Retromer-Dependent Recycling of MIG-14/Wntless in Wnt-Producing Cells. Dev Cell 2008;14:140-147.

51 Yang G, Xiao X, Rosen DG, et al. The biphasic role of $\mathrm{NF}-\mathrm{\kappa B}$ in progression and chemoresistance of ovarian cancer. Clin Cancer Res 2011;17:2181-2194.
52 Camp ER, Li J, Minnich DJ, et al. Inducible nuclear factor $-\kappa B$ activation contributes to chemotherapy resistance in gastric cancer. J Am Coll Surg 2004;199:249-258.

53 Biswas DK, Shi Q, Baily S, et al. NF- $\kappa$ B activation in human breast cancer specimens and its role in cell proliferation and apoptosis. Proc Natl Acad Sci USA 2004;101:10137-10142.

54 Ahmed K, Cao N, Li J. HER-2 and NF-кB as the targets for therapy-resistant breast cancer. Anticancer Res 2006;26:4235-4243.

55 McDermott SP, Wicha MS. Targeting breast cancer stem cells. Mol Oncol 2010;4:404-419.

56 de Hoon JPJ, Veeck J, BEPJ Vriens, et al. Tjan-Heijnen VCG. Taxane resistance in breast cancer: A closed HER2 circuit? Biochim Biophys Acta 2012;1825:197-206.

57 Zimmerman Z, Moon R, Chien A. Targetting Wnt pathways in disease. Cold Spring Harb Perspect Biol 2012;4:a008086.

58 Vogel CL, Cobleigh MA, Tripathy D, et al. Efficacy and safety of trastuzumab as a single agent in first-line treatment of HER2-overexpressing metastatic breast cancer. J Clin Oncol 2002;20:719-726.

59 Baselga J, Carbonell X, Castañeda-Soto N, et al. Phase II study of efficacy, safety, and pharmacokinetics of trastuzumab monotherapy administered on a 3-weekly schedule. J Clin Oncol 2005;23:2162-2171. 